MATHEMATICS OF COMPUTATION

Volume 80, Number 274, April 2011, Pages 887-904

S $0025-5718(2010) 02425-1$

Article electronically published on September 22, 2010

\title{
ON THE FAST COMPUTATION OF HIGH DIMENSIONAL VOLUME POTENTIALS
}

\author{
FLAVIA LANZARA, VLADIMIR MAZ'YA, AND GUNTHER SCHMIDT
}

\begin{abstract}
A fast method of an arbitrary high order for approximating volume potentials is proposed, which is effective also in high dimensional cases. Basis functions introduced in the theory of approximate approximations are used. Results of numerical experiments, which show approximation order $O\left(h^{8}\right)$ for the Newton potential in high dimensions, for example, for $n=200000$, are provided. The computation time scales linearly in the space dimension. New one-dimensional integral representations with separable integrands of the potentials of advection-diffusion and heat equations are obtained.
\end{abstract}

\section{INTRODUCTION}

The cubature of high-dimensional volume potentials plays an important role in a wide range of applications in physics, chemistry, biology, financial mathematics etc. Even a few years ago this problem encountered unsurmountable difficulties due to the so-called "curse of dimensionality". With the development of separated representations (also called tensor-structured approximations) by Beylkin and Mohlenkamp in 3, 4, the problem became tractable. In fact, in recent years several fast algorithms for the computation of multi-dimensional convolutions with singular kernels have been proposed; cf. [2, 6, 7, 8, 9, 5]. They are mainly based on piecewise polynomial approximations of a separated representation of the density. Then a suitable separated approximation of the action of the convolution operator on the basis functions allows one to determine the multi-dimensional convolution in question by computing a number of one-dimensional convolutions. In this way, the complexity of computing the multi-dimensional convolutions on a uniform tensor-product grid of size $h$ can be reduced from $O\left(h^{-n}|\log h|\right)$, achieved with the traditional multi-variate FFT, to $O\left(n h^{-1}|\log h|\right)$, where $n$ is the space dimension. A certain drawback of the schemes used in present is the necessity to find accurate separated representations of the convolution operator acting on piecewise polynomials. This procedure has been developed only for a few particular kernels $([2,7])$ and is rather involved, especially for higher order approximations.

In this article we propose a fast method of an arbitrary high order for approximating volume potentials, which is effective also in high dimensional cases. We use basis functions introduced in the theory of approximate approximations [11, 12]; see also 13 and the references therein. We report on numerical experiments which show

Received by the editor November 2, 2009 and, in revised form, February 20, 2010.

2010 Mathematics Subject Classification. Primary 65D32; Secondary 65-05.

This research was partially supported by the UK and Engineering and Physical Sciences Research Council via the grant EP/F005563/1. 
approximation order $O\left(h^{8}\right)$ for the Newton potential up to dimension $n=200000$. The computational complexity of the algorithm scales linearly in the physical dimension. In the experiments, high order cubatures lead to a minor increase of the computation time in comparison with second order approximations, but they are more accurate by several orders of magnitude, especially in high dimension.

The method combines our basis functions with an approach from Khoromskij 9 for computing volume potentials on uniform or composite refined grids. For brevity of exposition we restrict ourselves to the case of uniform grids with step size $h$. In [9], the density is approximated by piecewise constants and the action of the convolution operator on the basis function is written as a one-dimensional integral with a separable integrand, i.e., a product of functions depending only on one of the space variables. Then an accurate quadrature rule of this one-dimensional integral provides a separated representation of the convolution operator. The convergence orders $O\left(h^{2}\right)$ and $O\left(h^{3}\right)$ using Richardson extrapolation are confirmed by numerical experiments for $n=3$.

Instead of piecewise constants we use basis functions, which are Gaussians or products of Gaussians and special polynomials and give rise to high-order semianalytic cubature formulas for volume potentials. In Section 2 we describe these formulas and give error estimates. According to [14 (see also [13, Section 6.3]), the action of volume potentials on the basis functions allows for one-dimensional integral representations with separable integrands. In Section 3 we demonstrate this by the example of Newton's potential. We also obtain new one-dimensional representations for the potentials of the advection-diffusion and the heat equations. These one-dimensional integrals in combination with a quadrature rule lead to accurate separated representations of the potentials. In Section 4 , we provide results of numerical experiments for the Newton potential showing that even for very high space dimensions these approximations preserve the predicted convergence order of the cubature. Finally, in Section 5 we describe the quadrature of the one-dimensional integral representations used in the numerical examples. Here we also report on tests for second and fourth order formulas of the Newton potential and the inverse of $-\Delta+a^{2}$, which provide estimates of the rank of the separated representation required to approximate the action of the potential on a basis function with a prescribed relative error.

\section{Semi-ANALYTIC CUBAtURE FORMulas FOR POTENTIALS}

Here we collect some results on high-order cubature formulas for the volume potentials of the differential operators $-\Delta$ and $-\Delta+2 \mathbf{b} \cdot \nabla+c$ and of the heat potential in $\mathbb{R}^{n}$. More details can be found in [13].

2.1. Newton potentials in $\mathbb{R}^{n}$. The Newton potential is the inverse of the Laplace operator and given in $\mathbb{R}^{n}$ by the formula

$$
\mathcal{L}_{n} u(\mathbf{x})=\frac{\Gamma\left(\frac{n}{2}-1\right)}{4 \pi^{n / 2}} \int_{\mathbb{R}^{n}} \frac{u(\mathbf{y})}{|\mathbf{x}-\mathbf{y}|^{n-2}} d \mathbf{y}, \quad n \geq 3 .
$$

Here and in the following we denote by bold $\mathbf{x}$ a vector of length $n$ with the components $\mathbf{x}=\left(x_{1}, \ldots, x_{n}\right)$. The integral (2.1) is a unique solution of Poisson's equation

$$
-\Delta f=u \quad \text { in } \mathbb{R}^{n}, \quad|f(\mathbf{x})| \leq C|\mathbf{x}|^{n-2} \text { as }|\mathbf{x}| \rightarrow \infty .
$$


In [11, 12 cubature formulas for computing (2.1) were constructed which are based on the approximation of the density $u$ by functions with analytically known Newton potentials. In particular, one can approximate $u$ by the quasi-interpolant

$$
u_{h}(\mathbf{x})=\mathcal{D}^{-n / 2} \sum_{\mathbf{m} \in \mathbb{Z}^{n}} u(h \mathbf{m}) \eta_{2 M}\left(\frac{\mathbf{x}-h \mathbf{m}}{\sqrt{\overline{\mathcal{D}}} h}\right),
$$

with the basis function

$$
\eta_{2 M}(\mathbf{x})=\pi^{-n / 2} L_{M-1}^{(n / 2)}\left(|\mathbf{x}|^{2}\right) \mathrm{e}^{-|\mathbf{x}|^{2}}, \quad M \in \mathbb{N},
$$

where $L_{k}^{(\gamma)}$ are the generalized Laguerre polynomials

$$
L_{k}^{(\gamma)}(y)=\frac{\mathrm{e}^{y} y^{-\gamma}}{k !}\left(\frac{d}{d y}\right)^{k}\left(\mathrm{e}^{-y} y^{k+\gamma}\right), \quad \gamma>-1 .
$$

The Newton potential of $\eta_{2 M}$ is given by

$$
\mathcal{L}_{n} \eta_{2 M}(|\mathbf{x}|)=\frac{1}{4 \pi^{n / 2}|\mathbf{x}|^{n-2}} \gamma\left(\frac{n}{2}-1,|\mathbf{x}|^{2}\right)+\frac{\mathrm{e}^{-|\mathbf{x}|^{2}}}{\pi^{n / 2}} \sum_{j=0}^{M-2} \frac{L_{j}^{(n / 2-1)}\left(|\mathbf{x}|^{2}\right)}{4(j+1)}
$$

with the incomplete Gamma function

$$
\gamma(a, x)=\int_{0}^{x} \tau^{a-1} \mathrm{e}^{-\tau} d \tau
$$

Then the sum

$$
\mathcal{L}_{n} u_{h}(\mathbf{x})=\frac{h^{2}}{\mathcal{D}^{n / 2-1}} \sum_{\mathbf{m} \in \mathbb{Z}^{n}} u(h \mathbf{m}) \mathcal{L}_{n} \eta_{2 M}\left(\frac{\mathbf{x}-h \mathbf{m}}{\sqrt{\mathcal{D}} h}\right)
$$

is a semi-analytic cubature formula for $\mathcal{L}_{n} u$. It has been shown, that for sufficiently smooth and compactly supported functions (2.5) approximates $\mathcal{L}_{n} u$ with the error

$$
O\left(h^{2 M}\right)+O\left(\mathrm{e}^{-\mathcal{D} \pi^{2}} h^{2}\right) .
$$

This follows from the general asymptotic error estimate $O\left(h^{2 M}\right)+\varepsilon$ for the quasi-interpolant (2.2) with sufficiently smooth and decaying generating functions $\eta$ satisfying the moment condition

$$
\int_{\mathbb{R}^{n}} \eta(\mathbf{x}) d \mathbf{x}=1, \int_{\mathbb{R}^{n}} \mathbf{x}^{\boldsymbol{\alpha}} \eta(\mathbf{x}) d \mathbf{x}=0, \quad \forall \boldsymbol{\alpha}, 1 \leq|\boldsymbol{\alpha}|<2 M ;
$$

see [13, Chapter 2]. The saturation error $\varepsilon$ does not converge to zero as $h \rightarrow 0$, however, because of

$$
\varepsilon=O\left(\max _{\mathbf{x}}\left|\sum_{\mathbf{k} \in \mathbb{Z}^{n} \backslash\{\mathbf{0}\}} \mathcal{F} \eta(\sqrt{\mathcal{D}} \mathbf{k}) \mathrm{e}^{2 \pi i(\mathbf{k}, \mathbf{x}) / h}\right|\right),
$$

it can be made arbitrarily small if the parameter $\mathcal{D}$ is sufficiently large. Additionally, the Newton potential maps the fast oscillating saturation term (2.8) into a function with norm of order $O\left(h^{2} \varepsilon\right)$, which establishes the estimate (2.6). Therefore, in numerical computations with $\mathcal{D} \geq 3$ formula (2.5) behaves like a high order cubature formula. 
2.2. Potentials of advection-diffusion operators in $\mathbb{R}^{n}$. The fundamental solution of the operator $-\Delta+2 \mathbf{b} \cdot \nabla+c$ with a vector $\mathbf{b} \in \mathbb{C}^{n}$ and $c \in \mathbb{C}$ depends on the value of $c+|\mathbf{b}|^{2}$. Here we use the notation

$$
\langle\mathbf{y}, \mathbf{z}\rangle=\sum_{j=1}^{n} y_{j} z_{j} \quad \text { and } \quad|\mathbf{y}|^{2}=\langle\mathbf{y}, \mathbf{y}\rangle
$$

also for vectors $\mathbf{y}, \mathbf{z} \in \mathbb{C}^{n}$. If $c+|\mathbf{b}|^{2} \neq 0$, then the fundamental solution can be given as

$$
\kappa_{\lambda}(\mathbf{x})=\frac{\mathrm{e}^{\langle\mathbf{b}, \mathbf{x}\rangle}}{(2 \pi)^{n / 2}}\left(\frac{|\mathbf{x}|}{\lambda}\right)^{1-n / 2} K_{n / 2-1}(\lambda|\mathbf{x}|),
$$

where $\lambda \in \mathbb{C} \backslash(-\infty, 0]$ with $\lambda^{2}=c+|\mathbf{b}|^{2}$ and $K_{\nu}$ is the modified Bessel function of the second kind, also known as the Macdonald function, [1, 9.6]. If $c+|\mathbf{b}|^{2}=0$, then for $n \geq 3$,

$$
\kappa_{0}(\mathbf{x})=\frac{\Gamma\left(\frac{n}{2}-1\right)}{4 \pi^{n / 2}} \frac{\mathrm{e}^{\langle\mathbf{b}, \mathbf{x}\rangle}}{|\mathbf{x}|^{n-2}} .
$$

To derive a cubature formula for the volume potential with the kernel $\kappa_{\lambda}$ we look for a solution of

$$
-\Delta f+2 \mathbf{b} \cdot \nabla f+c f=\mathrm{e}^{-|\mathbf{x}|^{2}}, \mathbf{x} \in \mathbb{R}^{n},
$$

which is given as the one-dimensional integral

$$
f(\mathbf{x})=\frac{\mathrm{e}^{-|\mathbf{x}|^{2}} \lambda^{n / 2-1}}{|2 \mathbf{x}+\mathbf{b}|^{n / 2-1}} \int_{0}^{\infty} K_{n / 2-1}(\lambda r) I_{n / 2-1}(2|\mathbf{x}+\mathbf{b}| r) r \mathrm{e}^{-r^{2}} d r
$$

where $I_{\nu}$ is the modified Bessel function of the first kind; see [13, Section 5.2].

Using known analytic expressions of $I_{n+1 / 2}$ and $K_{n+1 / 2}$ (cf. [1]) it is possible to derive analytic formulas of (2.10) for odd space dimensions $n$. In particular, if $n=3$, then

$$
f(\mathbf{x})=\frac{\sqrt{\pi}}{4} \frac{\mathrm{e}^{-|\mathbf{x}|^{2}}}{|2 \mathbf{x}+\mathbf{b}|}\left(\mathrm{w}\left(\frac{i}{2}(\lambda-|2 \mathbf{x}+\mathbf{b}|)\right)-\mathrm{w}\left(\frac{i}{2}(\lambda+|2 \mathbf{x}+\mathbf{b}|)\right)\right),
$$

where $\mathrm{w}$ denotes the scaled complementary error function

$$
\mathrm{w}(z)=\mathrm{e}^{-z^{2}} \operatorname{erfc}(-i z)=\mathrm{e}^{-z^{2}}\left(1+\frac{2 i}{\sqrt{\pi}} \int_{0}^{z} \mathrm{e}^{t^{2}} d t\right)
$$

and erfc $=1-$ erf is the complementary error function. From

$$
L_{M-1}^{(n / 2)}\left(|\mathbf{x}|^{2}\right) \mathrm{e}^{-|\mathbf{x}|^{2}}=\sum_{k=0}^{M-1} \frac{(-1)^{k}}{k ! 4^{k}} \Delta^{k} \mathrm{e}^{-|\mathbf{x}|^{2}}
$$

(see [13, Theorem 3.5]), one can derive as in the case of Newton potentials semianalytic cubature formulas for the potential of the advection-diffusion equation with the approximation rate (2.6). 
2.3. Heat potential. Next we consider the non-homogeneous heat equation

$$
\begin{array}{rlrl}
f_{t}-\nu \Delta_{\mathbf{x}} f & =u(\mathbf{x}, t) & t \geq 0, \nu>0, \\
f(\mathbf{x}, 0) & =0 & \mathbf{x} \in \mathbb{R}^{n} .
\end{array}
$$

It is well known that the solution of this Cauchy problem can be written as

$$
f(\mathbf{x}, t)=\int_{0}^{t}\left(\mathcal{P}_{t-\lambda} u(\cdot, \lambda)\right)(\mathbf{x}) d \lambda,
$$

where $\mathcal{P}_{t}$ is the Poisson integral

$$
\left(\mathcal{P}_{t} u(\cdot, \lambda)\right)(\mathbf{x})=\frac{1}{(4 \pi \nu t)^{n / 2}} \int_{\mathbb{R}^{n}} \mathrm{e}^{-|\mathbf{x}-\mathbf{y}|^{2} /(4 \nu t)} u(\mathbf{y}, \lambda) d \mathbf{y} .
$$

Suppose that the right-hand side $u$ is a sufficiently smooth function with compact support in $\mathbb{R}^{n} \times \mathbb{R}_{+}$. An approximation of the solution $f(\mathbf{x}, t)$ of $(2.12)$ can be obtained if $u$ is approximated by the quasi-interpolant on the rectangular grid $\{(h \mathbf{m}, \tau i)\}$

$$
u_{h, \tau}(\mathbf{x}, t)=\frac{\pi^{-(n+1) / 2}}{\sqrt{\mathcal{D}_{0} \mathcal{D}^{n}}} \sum_{\substack{i \in \mathbb{Z} \\ \mathbf{m} \in \mathbb{Z}^{n}}} u(h \mathbf{m}, \tau i) \mathrm{e}^{-(t-\tau i)^{2} /\left(\mathcal{D}_{0} \tau^{2}\right)} \mathrm{e}^{-|\mathbf{x}-h \mathbf{m}|^{2} /\left(\mathcal{D} h^{2}\right)} .
$$

It can be easily seen, that

$$
\begin{aligned}
& \left(\mathcal{P}_{t-\lambda} u_{h, \tau}(\cdot, \lambda)\right)(\mathbf{x}) \\
& \quad=\frac{h^{n}}{\pi^{n / 2} \sqrt{\pi \mathcal{D}_{0}}} \sum_{\substack{i \in \mathbb{Z} \\
\mathbf{m} \in \mathbb{Z}^{n}}} u(h \mathbf{m}, \tau i) \frac{\mathrm{e}^{-(\lambda-\tau i)^{2} /\left(\mathcal{D}_{0} \tau^{2}\right)} \mathrm{e}^{-|\mathbf{x}-h \mathbf{m}|^{2} /\left(\mathcal{D} h^{2}+4 \nu(t-\lambda)\right)}}{\left(\mathcal{D} h^{2}+4 \nu(t-\lambda)\right)^{n / 2}},
\end{aligned}
$$

hence the sum

$$
\begin{aligned}
f_{h, \tau}(\mathbf{x}, t) & =\int_{0}^{t}\left(\mathcal{P}_{t-\lambda} u_{h, \tau}(\cdot, \lambda)\right)(\mathbf{x}) d \lambda \\
& =\frac{h^{n}}{\pi^{n / 2} \sqrt{\pi \mathcal{D}_{0}}} \sum_{\substack{i \in \mathbb{Z} \\
\mathbf{m} \in \mathbb{Z}^{n}}} K_{2}(\mathbf{x}-h \mathbf{m}, t, \tau i) u(h \mathbf{m}, \tau i)
\end{aligned}
$$

is an approximation of $f(\mathbf{x}, t)$, where we use the notation

$$
K_{2}(\mathbf{x}, t, \tau i)=\int_{0}^{t} \frac{\mathrm{e}^{-(t-\lambda-\tau i)^{2} /\left(\mathcal{D}_{0} \tau^{2}\right)} \mathrm{e}^{-|\mathbf{x}|^{2} /\left(\mathcal{D} h^{2}+4 \nu \lambda\right)}}{\left(\mathcal{D} h^{2}+4 \nu \lambda\right)^{n / 2}} d \lambda .
$$

Since $u_{h, \tau}(\mathbf{x}, t)$ differs from $u(\mathbf{x}, t)$ by

$$
\left|u(\mathbf{x}, t)-u_{h, \tau}(\mathbf{x}, t)\right| \leq \varepsilon+c\left(\left(\tau \sqrt{\mathcal{D}_{0}}\right)^{2}+(h \sqrt{\mathcal{D}})^{2}\right), \quad \forall \mathbf{x} \in \mathbb{R}^{n}, t \in[0, T],
$$


the function $f_{h, \tau}(\mathbf{x}, t)$ approximates the solution $f(\mathbf{x}, t)$ with the error

$$
\begin{aligned}
\mid f(\mathbf{x}, t) & -f_{h, \tau}(\mathbf{x}, t) \mid \\
& =\frac{1}{(4 \pi \nu)^{n / 2}} \int_{0}^{t} d \lambda \int_{\mathbb{R}^{n}} \frac{\mathrm{e}^{-|\mathbf{x}-\mathbf{y}|^{2} /(4(t-\lambda))}}{(t-\lambda)^{n / 2}}\left|u(\mathbf{y}, \lambda)-u_{h, \tau}(\mathbf{y}, \lambda)\right| d \mathbf{y} \\
& \leq T\left\|u-u_{h, \tau}\right\|_{L^{\infty}\left(\mathbb{R}^{n} \times[0, T]\right)} \leq \varepsilon+c\left(\left(\tau \sqrt{\mathcal{D}_{0}}\right)^{2}+(h \sqrt{\mathcal{D}})^{2}\right)
\end{aligned}
$$

for all $\mathbf{x} \in \mathbb{R}^{n}$ and $t \in[0, T]$.

\section{ONE-DIMENSIONAL INTEGRAL REPRESENTATIONS OF THE POTENTIALS ACTING ON GAUSSIANS}

The computation of the mentioned cubature formulas on the grid $\{h \mathbf{k}\}$ leads to discrete convolutions

$$
\sum_{\mathbf{m}} a_{\mathbf{k}-\mathbf{m}} u(h \mathbf{m}),
$$

where the indices $\mathbf{m}, \mathbf{k}$ belong to some subset of $\mathbb{Z}^{n}$, and the coefficients $a_{\mathbf{k}}$ are given either analytically or by a one-dimensional integral with smooth integrand. For general functions $u$ the most efficient summation methods for (3.1) are probably fast convolutions based on multi-variate FFT. However, even for the space dimension $n=3$ problems of moderate size often exceed the capacity of available computer systems.

The situation in high dimensions is much better when the vectors $\{u(h \mathbf{m})\}$ and $\left\{a_{\mathbf{k}}\right\}$ allow separated representations [3], i.e., for given accuracy $\epsilon$ they can be represented as a sum of products of vectors in dimension 1

$$
\begin{aligned}
& u\left(h m_{1}, \ldots, h m_{n}\right)=\sum_{p=1}^{R} r_{p} \prod_{j=1}^{n} u_{j}^{(p)}\left(h m_{j}\right)+O(\epsilon), \\
& a\left(k_{1}, \ldots, k_{n}\right)=\sum_{p=1}^{R} s_{p} \prod_{j=1}^{n} v_{j}^{(p)}\left(k_{j}\right)+O(\epsilon) .
\end{aligned}
$$

Then an approximate value of (3.1) can be computed by the sum of products of one-dimensional convolutions

$$
\sum_{\mathbf{m}} a_{\mathbf{k}-\mathbf{m}} u(h \mathbf{m}) \approx \sum_{p, q=1}^{R} r_{p} s_{q} \prod_{j=1}^{n} \sum_{m_{j}} v_{j}^{(q)}\left(k_{j}-m_{j}\right) u_{j}^{(p)}\left(h m_{j}\right) .
$$

To obtain a separated representation of the vector $\left\{a_{\mathbf{k}}\right\}$ the following idea from Khoromskij 9] can be applied: Suppose that the density $u$ is approximated by interpolation or quasi-interpolation using linear combinations of the translates $\phi(\cdot-$ $h \mathbf{m})$ of a basis function. Then the components of $\left\{a_{\mathbf{k}}\right\}$ are the values of the volume potential with the kernel $g$ acting on $\phi$,

$$
a_{\mathbf{k}}=\int_{\mathbb{R}^{n}} g(\mathbf{k}-\mathbf{y}) \phi(\mathbf{y}) d \mathbf{y} .
$$


If this integral can be transformed to a one-dimensional integral with separable integrand

$$
\int_{\mathbb{R}^{n}} g(\mathbf{x}-\mathbf{y}) \phi(\mathbf{y}) d \mathbf{y}=\int_{0}^{\infty} \prod_{j=1}^{n} g_{j}\left(x_{j}, t\right) d t
$$

then a separated approximation of $\left\{a_{\mathbf{k}}\right\}$ is obtained by applying an accurate quadrature rule for this integral,

$$
a_{\mathbf{k}} \approx \sum_{\ell=1}^{R} \omega_{\ell} \prod_{j=1}^{n} g_{j}\left(k_{j}, \tau_{\ell}\right) .
$$

In 9] one-dimensional integral representations were obtained for the potentials of the Laplace and of the advection-diffusion equation with $\mathbf{b}=0, c>0$, applied to the characteristic functions $\phi$ of a cube in $\mathbb{R}^{3}$. This gives rise to a fast algorithm which is based on piecewise constant basis functions. The convergence order $O\left(h^{2}\right)$ is proved together with the order $O\left(h^{3}\right)$ by using Richardson extrapolation.

In this section we describe one-dimensional integral representations with separable integrands for the Newton potential and the potentials of the advection-diffusion and the heat equations acting on basis functions introduced in the previous section.

3.1. Newton potential. The separated representation of the second order cubature formula

$$
\frac{\mathcal{D} h^{2}}{(\pi \mathcal{D})^{n / 2}} \sum_{\mathbf{m} \in \mathbb{Z}^{n}} u(h \mathbf{m}) \mathcal{L}_{n}\left(\mathrm{e}^{-|\cdot|^{2}}\right)\left(\mathbf{r}_{\mathbf{m}}\right)
$$

for the Newton potential with

$$
\mathbf{r}_{\mathbf{m}}=\frac{\mathbf{x}-h \mathbf{m}}{\sqrt{\mathcal{D}} h} \quad \text { and } \quad \mathcal{L}_{n}\left(\mathrm{e}^{-|\cdot|^{2}}\right)(\mathbf{x})=\frac{1}{4|\mathbf{x}|^{n-2}} \gamma\left(\frac{n}{2}-1,|\mathbf{x}|^{2}\right),
$$

follows from the formula

$$
\mathcal{L}_{n}\left(\mathrm{e}^{-|\cdot|^{2}}\right)(\mathbf{x})=\frac{1}{4} \int_{0}^{\infty} \frac{\mathrm{e}^{-|\mathbf{x}|^{2} /(1+t)}}{(1+t)^{n / 2}} d t
$$

which was obtained in [14] and is valid for $n \geq 3$. The quadrature rule of the last integral

$$
\mathcal{L}_{n}\left(\mathrm{e}^{-|\cdot|^{2}}\right)(\mathbf{x}) \approx \frac{1}{4} \sum_{p=1}^{R} \omega_{p} \frac{\mathrm{e}^{-|\mathbf{x}|^{2} /\left(1+\tau_{p}\right)}}{\left(1+\tau_{p}\right)^{n / 2}}=\frac{1}{4} \sum_{p=1}^{R} \omega_{p} \prod_{j=1}^{n} \frac{\mathrm{e}^{-x_{j}^{2} /\left(1+\tau_{p}\right)}}{\left(1+\tau_{p}\right)^{1 / 2}}
$$

with certain quadrature weights $\omega_{p}$ and nodes $\tau_{p}$ gives the separated approximation

$$
\mathcal{L}_{n} u_{h}(h \mathbf{k}) \approx \frac{\mathcal{D} h^{2}}{(\pi \mathcal{D})^{n / 2}} \sum_{\mathbf{m} \in \mathbb{Z}^{n}} u(h \mathbf{m}) \sum_{k=1}^{R} \omega_{k} \prod_{j=1}^{n} \frac{\mathrm{e}^{-\left(k_{j}-m_{j}\right)^{2} /\left(\mathcal{D}\left(1+\tau_{k}\right)\right)}}{\left(1+\tau_{k}\right)^{1 / 2}} .
$$

To get an approximation for higher order cubature formulas we note that the same convergence order $O\left(h^{2 M}\right)+O\left(\mathrm{e}^{-\mathcal{D} \pi^{2}} h^{2}\right)$ as in the case of generating functions (2.3) holds, when the density is approximated by the sum

$$
\mathcal{M}_{M} u(\mathbf{x})=(\pi \mathcal{D})^{-n / 2} \sum_{\mathbf{m} \in \mathbb{Z}^{n}} u(h \mathbf{m}) \prod_{j=1}^{n} \widetilde{\eta}_{2 M}\left(\frac{x_{j}-h m_{j}}{\sqrt{\mathcal{D}} h}\right) .
$$


Here the basis function is the tensor product of the one-dimensional functions

$$
\widetilde{\eta}_{2 M}(x)=L_{M-1}^{(1 / 2)}\left(x^{2}\right) \mathrm{e}^{-x^{2}},
$$

which obviously satisfies the moment condition (2.7). Thus the cubature formula

$$
\mathcal{L}_{M, h}^{(n)} u(\mathbf{x})=\frac{h^{2}}{\mathcal{D}^{n / 2-1}} \sum_{\mathbf{m} \in \mathbb{Z}^{n}} u(h \mathbf{m}) \mathcal{L}_{n}\left(\prod_{j=1}^{n} \widetilde{\eta}_{2 M}\right)\left(\frac{\mathbf{x}-h \mathbf{m}}{\sqrt{\mathcal{D}} h}\right)
$$

approximates $\mathcal{L}_{n} u$ with the error (2.6). The relation

$$
\widetilde{\eta}_{2 M}(x)=\sum_{k=0}^{M-1} \frac{(-1)^{k}}{k ! 4^{k}} \frac{d^{2 k}}{d x^{2 k}} \mathrm{e}^{-x^{2}}=\mathrm{e}^{-x^{2}} \sum_{k=0}^{M-1} L_{k}^{(-1 / 2)}\left(x^{2}\right),
$$

leads to the one-dimensional integral representation of $\mathcal{L}_{n}\left(\prod_{j=1}^{n} \widetilde{\eta}_{2 M}\right)$ by writing the solution of the Poisson equation

$$
-\Delta u(\mathbf{x})=\prod_{j=1}^{n} \widetilde{\eta}_{2 M}\left(x_{j}\right)
$$

as the integral

$$
\begin{aligned}
u(\mathbf{x}) & =\frac{1}{4} \prod_{j=1}^{n} \sum_{k=0}^{M-1} \frac{(-1)^{k}}{k ! 4^{k}} \frac{d^{2 k}}{d x_{j}^{2 k}} \int_{0}^{\infty} \frac{\mathrm{e}^{-|\mathbf{x}|^{2} /(1+t)}}{(1+t)^{1 / 2}} d t \\
& =\frac{1}{4} \int_{0}^{\infty}\left(\prod_{j=1}^{n} \sum_{k=0}^{M-1} \frac{(-1)^{k}}{k ! 4^{k}} \frac{d^{2 k}}{d x_{j}^{2 k}} \mathrm{e}^{-x_{j}^{2} /(1+t)}\right) \frac{d t}{(1+t)^{n / 2}} \\
& =\frac{1}{4} \int_{0}^{\infty}\left(\prod_{j=1}^{n} \sum_{k=0}^{M-1} \frac{\mathrm{e}^{-x_{j}^{2} /(1+t)}}{(1+t)^{k}} L_{k}^{(-1 / 2)}\left(\frac{x_{j}^{2}}{1+t}\right)\right) \frac{d t}{(1+t)^{n / 2}} .
\end{aligned}
$$

Hence the separated representation of the Newton potential is reduced to find accurate quadrature rules for the parameter dependent integrals

$$
I_{M}(\mathbf{x})=\int_{0}^{\infty}\left(\prod_{j=1}^{n} \mathrm{e}^{-x_{j}^{2} /(1+t)} \sum_{k=0}^{M-1} \frac{1}{(1+t)^{k}} L_{k}^{(-1 / 2)}\left(\frac{x_{j}^{2}}{1+t}\right)\right) \frac{d t}{(1+t)^{n / 2}}, M \geq 1 .
$$

It is necessary that the quadrature rule approximates the integrals (3.6) with prescribed error for the parameters $x_{j}=\left(k_{j}-m_{j}\right) / \sqrt{\mathcal{D}}$ within the range $\left|x_{j}\right| \leq K$ and some given bound $K$. This will be discussed in Section 5 .

3.2. Potentials of advection-diffusion operators. Here we look for a onedimensional integral representation with separable integrand of the integral (2.10). Let $f$ be the solution of the advection-diffusion equation (2.9). Then $v=f \mathrm{e}^{-\langle\mathbf{b}, \mathbf{x}\rangle}$ satisfies

$$
-\Delta v+\lambda^{2} v=\mathrm{e}^{-|\mathbf{x}+\mathbf{b} / 2|^{2}} \mathrm{e}^{|\mathbf{b}|^{2} / 4} \quad \text { with } \lambda^{2}=c+|\mathbf{b}|^{2}
$$

in $\mathbb{R}^{n}$. In was shown in 14 that $v$ can be represented in the form

$$
v(\mathbf{x})=\frac{\mathrm{e}^{|\mathbf{b}|^{2} / 4}}{4} \int_{0}^{\infty} \frac{\mathrm{e}^{-\lambda^{2} t / 4} \mathrm{e}^{-|\mathbf{x}+\mathbf{b} / 2|^{2} /(1+t)}}{(1+t)^{n / 2}} d t
$$


for $\operatorname{Re} \lambda^{2} \geq 0$ and $n \geq 3$. If $\operatorname{Re} \lambda^{2}>0$, then (3.7) is valid for all space dimensions $n$; see also [13, Theorem 6.4]. Thus

$$
\begin{aligned}
f(\mathbf{x}) & =\int_{\mathbb{R}^{n}} \kappa_{\lambda}(\mathbf{x}-\mathbf{y}) \mathrm{e}^{-|\mathbf{y}|^{2}} d \mathbf{y}=\frac{\mathrm{e}^{\langle\mathbf{b}, \mathbf{x}\rangle} \mathrm{e}^{|\mathbf{b}|^{2} / 4}}{4} \int_{0}^{\infty} \frac{\mathrm{e}^{-t\left(c+|\mathbf{b}|^{2}\right) / 4} \mathrm{e}^{-|\mathbf{x}+\mathbf{b} / 2|^{2} /(1+t)}}{(1+t)^{n / 2}} d t \\
(3.8) & =\frac{1}{2} \int_{0}^{\infty} \frac{\mathrm{e}^{-c t / 2}}{(1+2 t)^{n / 2}} \mathrm{e}^{-|\mathbf{x}-t \mathbf{b}|^{2} /(1+2 t)} d t .
\end{aligned}
$$

Consequently, if $\operatorname{Re}\left(c+|\mathbf{b}|^{2}\right) \geq 0$, then an approximate solution of the advectiondiffusion equation

$$
-\Delta f+2 \mathbf{b} \cdot \nabla f+c f=u
$$

is given in $\mathbb{R}^{n}$ by the sum

$$
f_{h}(\mathbf{x})=\frac{\mathcal{D} h^{2}}{2(\pi \mathcal{D})^{n / 2}} \sum_{\mathbf{m} \in \mathbb{Z}^{n}} u(h \mathbf{m}) \int_{0}^{\infty} \frac{\mathrm{e}^{-\mathcal{D} h^{2} c t / 2}}{(1+2 t)^{n / 2}} \mathrm{e}^{-\left|\mathbf{x}-h \mathbf{m}-t \mathcal{D} h^{2} \mathbf{b}\right|^{2} /\left(\mathcal{D} h^{2}(1+2 t)\right)} d t,
$$

which approximates $f$ with the order $O\left(h^{2}\right)$. Analogously to the case of Newton potentials the one-dimensional integrals for cubature formulas of order (2.6) are based on the basis functions $\prod \widetilde{\eta}_{2 M}\left(x_{j}\right)$. Now the relation (3.5) leads to

$$
\begin{aligned}
\int_{\mathbb{R}^{n}} \kappa_{\lambda}(\mathbf{x}-\mathbf{y}) & \prod_{j=1}^{n} \widetilde{\eta}_{2 M}\left(y_{j}\right) d \mathbf{y}=\prod_{j=1}^{n} \sum_{k=0}^{M-1} \frac{(-1)^{k}}{k ! 4^{k}} \frac{\partial^{2 k}}{\partial x_{j}^{2 k}} \int_{\mathbb{R}^{n}} \kappa_{\lambda}(\mathbf{x}-\mathbf{y}) \mathrm{e}^{-|\mathbf{y}|^{2}} d \mathbf{y} \\
& =\frac{1}{2} \int_{0}^{\infty} \frac{\mathrm{e}^{-c t / 2}}{(1+2 t)^{n / 2}}\left(\prod_{j=1}^{n} \sum_{k=0}^{M-1} \frac{(-1)^{k}}{k ! 4^{k}} \frac{d^{2 k}}{d x_{j}^{2 k}} \mathrm{e}^{-|\mathbf{x}-t \mathbf{b}|^{2} /(1+2 t)}\right) d t \\
& =\frac{1}{2} \int_{0}^{\infty} \frac{\mathrm{e}^{-c t / 2}}{(1+2 t)^{n / 2}}\left(\prod_{j=1}^{n} g_{M}\left(2 t, x_{j}-t b_{j}\right)\right) d t
\end{aligned}
$$

with the function

$$
g_{M}(t, x)=\sum_{k=0}^{M-1} \frac{\mathrm{e}^{-x^{2} /(1+t)}}{(1+t)^{k}} L_{k}^{(-1 / 2)}\left(\frac{x^{2}}{1+t}\right) .
$$

3.3. Heat potential. Recall that the approximation of the non-homogeneous heat equation (2.12) on the rectangular grid $\{(h \mathbf{k}, \tau \ell)\}$ is given by the $(n+1)$-dimensional convolution

$$
f_{h, \tau}(h \mathbf{k}, \tau \ell)=\frac{h^{n}}{\pi^{(n+1) / 2} \sqrt{\mathcal{D}_{0}}} \sum_{\substack{i \in \mathbb{Z} \\ \mathbf{m} \in \mathbb{Z}^{n}}} K_{2}(h(\mathbf{k}-\mathbf{m}), \tau \ell, \tau i) u(h \mathbf{m}, \tau i),
$$

where the integral $K_{2}$ given by (2.14) cannot be taken analytically. Making the substitution,

$$
\lambda=\frac{t}{1+\mathrm{e}^{-\xi}},
$$

$K_{2}(\mathbf{x}, t, \tau i)$ transforms to the integral over $\mathbb{R}$,

$$
K_{2}(\mathbf{x}, t, \tau i)=\frac{t}{4} \int_{-\infty}^{\infty} \frac{\mathrm{e}^{-\left(t /\left(1+\mathrm{e}^{\xi}\right)-\tau i\right)^{2} /\left(\mathcal{D}_{0} \tau^{2}\right)} \mathrm{e}^{-|\mathbf{x}|^{2} /\left(\mathcal{D} h^{2}+4 \nu t /\left(1+\mathrm{e}^{-\xi}\right)\right)}}{\left(\mathcal{D} h^{2}+4 \nu t /\left(1+\mathrm{e}^{-\xi}\right)\right)^{n / 2} \cosh ^{2}(\xi / 2)} d \xi
$$


with exponentially decaying integrand. The quadrature rule for $K_{2}(h \mathbf{k}, \tau \ell, \tau i)$,

$$
\frac{\tau \ell}{4} \sum_{p=1}^{R} \omega_{p} \frac{\mathrm{e}^{-\left(\ell /\left(1+\mathrm{e}^{\xi_{p}}\right)-i\right)^{2} / \mathcal{D}_{0}}}{\left(\mathcal{D} h^{2}+4 \nu \tau \ell /\left(1+\mathrm{e}^{-\xi_{p}}\right)\right)^{n / 2} \cosh ^{2}\left(\xi_{p} / 2\right)} \prod_{j=1}^{n} \mathrm{e}^{-k_{j}^{2} /\left(\mathcal{D}+4 \nu \tau \ell /\left(h^{2}\left(1+\mathrm{e}^{-\xi_{p}}\right)\right)\right)},
$$

with certain weights $\omega_{p}$ and nodes $\xi_{p}$ and the separated representation of the righthand side of (2.12),

$$
u(h \mathbf{x}, t)=\sum_{q=1}^{Q} r_{q} \prod_{j=1}^{n} u_{j}^{(q)}\left(x_{j}, t\right)+O(\epsilon),
$$

leads to the approximation

$$
f_{h, \tau}(h \mathbf{k}, \tau \ell) \approx \frac{h^{n} \tau \ell}{4 \pi^{(n+1) / 2} \sqrt{\mathcal{D}_{0}}} \sum_{q=1}^{Q} \sum_{p=1}^{R} \frac{\omega_{p} r_{q}}{\cosh ^{2}\left(\xi_{p} / 2\right)} \sum_{i \in \mathbb{Z}} a_{\ell, i, p} c_{\mathbf{k}, \ell, i, p}^{(q)},
$$

where we use the abbreviation

$$
a_{\ell, i, p}=\frac{\mathrm{e}^{-\left(\ell /\left(1+\mathrm{e}^{\xi_{p}}\right)-i\right)^{2} / \mathcal{D}_{0}}}{\left(\mathcal{D} h^{2}+4 \nu \tau \ell /\left(1+\mathrm{e}^{-\xi_{p}}\right)\right)^{n / 2}},
$$

and $c_{\mathbf{k}, \ell, i, p}^{(q)}$ is the product of one-dimensional convolutions

$$
c_{\mathbf{k}, \ell, i, p}^{(q)}=\prod_{j=1}^{n} \sum_{m_{j} \in \mathbb{Z}} u_{j}^{(q)}\left(h m_{j}, \tau i\right) \mathrm{e}^{-\left(k_{j}-m_{j}\right)^{2} /\left(\mathcal{D}+4 \nu \tau \ell /\left(h^{2}\left(1+\mathrm{e}^{-\xi_{p}}\right)\right)\right)} .
$$

Approximations which converge with higher order to the solution of (2.12) can be obtained if the right-hand side $u$ is approximated by

$$
u_{h, \tau}(\mathbf{x}, t)=\frac{\pi^{-(n+1) / 2}}{\sqrt{\mathcal{D}_{0} \mathcal{D}^{n}}} \sum_{\substack{i \in \mathbb{Z} \\ \mathbf{m} \in \mathbb{Z}^{n}}} u(h \mathbf{m}, \tau i) \widetilde{\eta}_{2 S}\left(\frac{t-\tau i}{\sqrt{\mathcal{D}_{0}} \tau}\right) \prod_{j=1}^{n} \widetilde{\eta}_{2 M}\left(\frac{x_{j}-h m_{j}}{\sqrt{\mathcal{D}} h}\right)
$$

with the basis functions $\widetilde{\eta}_{2 M}$ defined by (3.3). Then by using (3.5) the heat potential of the quasi-interpolant (3.10) is given by

$$
f_{h, \tau}(\mathbf{x}, t)=\frac{h^{n}}{\pi^{n / 2} \sqrt{\pi \mathcal{D}_{0}}} \sum_{\substack{i \in \mathbb{Z} \\ \mathbf{m} \in \mathbb{Z}^{n}}} K_{S, M}(\mathbf{x}-h \mathbf{m}, t, \tau i) u(h \mathbf{m}, \tau i),
$$

where we denote

$$
K_{S, M}(\mathbf{x}, t, \tau i)=\sum_{k=0}^{S-1} \frac{(-1)^{k}\left(\mathcal{D}_{0} \tau^{2}\right)^{k}}{k ! 4^{k}} \frac{\partial^{2 k}}{\partial t^{2 k}} \prod_{j=1}^{n} \sum_{\ell=0}^{M-1} \frac{(-1)^{\ell}\left(\mathcal{D} h^{2}\right)^{\ell}}{\ell ! 4^{\ell}} \frac{\partial^{2 \ell}}{\partial x_{j}^{2 \ell}} K_{2}(\mathbf{x}, t, \tau i) .
$$

We have

$$
\frac{(-1)^{\ell}}{\ell ! 4^{\ell}} \frac{\partial^{2 \ell}}{\partial x_{j}^{2 \ell}} \mathrm{e}^{-|\mathbf{x}-h \mathbf{m}|^{2} /\left(\mathcal{D} h^{2}+4 \nu \lambda\right)}=\frac{\mathrm{e}^{-|\mathbf{x}-h \mathbf{m}|^{2} /\left(\mathcal{D} h^{2}+4 \nu \lambda\right)}}{\left(\mathcal{D} h^{2}+4 \nu \lambda\right)^{\ell}} L_{\ell}^{(-1 / 2)}\left(\frac{\left(x_{j}-h m_{j}\right)^{2}}{\mathcal{D} h^{2}+4 \nu \lambda}\right),
$$

which by using (2.14) leads to the representation with separable integrand

$$
K_{S, M}(\mathbf{x}, t, \tau i)=\sum_{k=0}^{S-1} \frac{(-1)^{k}\left(\mathcal{D}_{0} \tau^{2}\right)^{k}}{k ! 4^{k}} \frac{\partial^{2 k}}{\partial t^{2 k}} \int_{0}^{t} \mathrm{e}^{-(\lambda-(t-\tau i))^{2} /\left(\mathcal{D}_{0} \tau^{2}\right)} \prod_{j=1}^{n} g_{M}\left(\lambda, x_{j}\right) d \lambda .
$$


Here we denote by $g_{M}$ the function

$$
g_{M}(\lambda, x)=\mathrm{e}^{-x^{2} /\left(\mathcal{D} h^{2}+4 \nu \lambda\right)} \sum_{\ell=0}^{M-1} \frac{\left(\mathcal{D} h^{2}\right)^{\ell}}{\left(\mathcal{D} h^{2}+4 \nu \lambda\right)^{\ell+1 / 2}} L_{\ell}^{(-1 / 2)}\left(\frac{x^{2}}{\mathcal{D} h^{2}+4 \nu \lambda}\right) .
$$

\section{Numerical RESUlts for the NeWton POtential}

We provide results of some experiments which show the accuracy and numerical convergence orders of the method. In the cube $[-6,6]^{n}, n \geq 3$, we compute the Newton potential of the densities

$$
u_{1}(\mathbf{x})=\mathrm{e}^{-|\mathbf{x}|^{2}} \quad \text { and } \quad u_{2}(\mathbf{x})=\left(4|\mathbf{x}|^{2}-2 n\right) \mathrm{e}^{-|\mathbf{x}|^{2}},
$$

which have the exact values

$$
\mathcal{L}_{n} u_{1}(\mathbf{x})=\frac{1}{4|\mathbf{x}|^{n-2}} \gamma\left(\frac{n}{2}-1,|\mathbf{x}|^{2}\right) \quad \text { and } \quad \mathcal{L}_{n} u_{2}(\mathbf{x})=-\mathrm{e}^{-|\mathbf{x}|^{2}} .
$$

TABLE 1. Exact and approximated values of $\mathcal{L}_{n} u_{1}\left(x_{1}, 0, \ldots, 0\right)$ and the relative error using $\mathcal{L}_{4,0.05}^{(n)}$

\begin{tabular}{l|l|cc|r} 
dimension & $x_{1}$ & exact & approximation & relative error \\
\hline \multirow{5}{*}{$n=3$} & 0.0 & 0.50000000000000 & 0.49999999923850 & $1.5230 \mathrm{E}-09$ \\
& 1.0 & 0.37341206640621 & 0.37341206614375 & $7.0287 \mathrm{E}-10$ \\
& 2.0 & 0.22052034769061 & 0.22052034766043 & $1.3685 \mathrm{E}-10$ \\
& 3.0 & 0.14770122470992 & 0.14770122470423 & $3.8549 \mathrm{E}-11$ \\
& 4.0 & 0.11077836397370 & 0.11077836396658 & $6.4242 \mathrm{E}-11$ \\
& 5.0 & 0.08862269254514 & 0.08862269253834 & $7.6764 \mathrm{E}-11$ \\
\hline \hline \multirow{5}{*}{$n=10$} & 0.0 & 0.06250000000000 & 0.06249999932963 & $1.0726 \mathrm{E}-08$ \\
& 1.0 & 0.02848223531423 & 0.02848223504590 & $9.4209 \mathrm{E}-09$ \\
& 2.0 & 0.00331951101348 & 0.00331951099712 & $4.9280 \mathrm{E}-09$ \\
& 3.0 & 0.00022377080789 & 0.00022377080851 & $2.7741 \mathrm{E}-09$ \\
& 4.0 & 0.00002288605175 & 0.00002288605169 & $2.6127 \mathrm{E}-09$ \\
& 5.0 & 0.00000383999984 & 0.00000383999985 & $6.8146 \mathrm{E}-10$ \\
\hline \hline \multirow{5}{*}{$n=100$} & 0.0 & 0.00510204081633 & 0.00510204386664 & $5.9786 \mathrm{E}-07$ \\
& 1.0 & 0.00191522512312 & 0.00191522620272 & $5.6369 \mathrm{E}-07$ \\
& 2.0 & 0.00010155802170 & 0.00010155808096 & $5.8347 \mathrm{E}-07$ \\
& 3.0 & 0.00000076714427 & 0.00000076714503 & $9.9929 \mathrm{E}-07$ \\
& 4.0 & 0.00000000084085 & 0.00000000084085 & $1.8801 \mathrm{E}-06$ \\
& 5.0 & 0.00000000000014 & 0.00000000000014 & $3.6702 \mathrm{E}-05$ \\
\hline \hline \multirow{3}{*}{$n=300$} & 0.0 & 0.00167785234899 & 0.00167786399030 & $6.9382 \mathrm{E}-06$ \\
& 1.0 & 0.00062138979909 & 0.00062139403983 & $6.8246 \mathrm{E}-06$ \\
& 2.0 & 0.00003157272440 & 0.00003157294168 & $6.8819 \mathrm{E}-06$ \\
& 3.0 & 0.00000022027431 & 0.00000022027615 & $8.3417 \mathrm{E}-06$ \\
& 4.0 & 0.00000000021134 & 0.00000000021134 & $8.4873 \mathrm{E}-06$ \\
& 5.0 & 0.00000000000003 & 0.00000000000003 & $2.6541 \mathrm{E}-05$ \\
\hline & & & & \\
\hline
\end{tabular}

In Table 1 we compare the values of the exact and the approximate solution for $\mathcal{L}_{n} u_{1}$ at some points $\left(x_{1}, 0, \ldots, 0\right) \in \mathbb{R}^{n}$ of the grid, where we have chosen the space dimensions $n=3,10,100,300$.

The approximations have been computed on a uniform grid with step size $h=$ 0.05 using the basis functions

$$
\widetilde{\eta}_{8}(\mathbf{x})=(\pi \mathcal{D})^{-n / 2} \mathrm{e}^{-|\mathbf{x}|^{2} / \mathcal{D}} \prod_{j=1}^{n} L_{3}^{(1 / 2)}\left(x_{j}^{2} / \mathcal{D}\right) \quad \text { with } \quad \mathcal{D}=3.5 .
$$


In this case one has to determine that

$$
I_{3}\left(\frac{\mathbf{m}}{\sqrt{\mathcal{D}}}\right)=\frac{1}{4} \int_{0}^{\infty}\left(\prod_{j=1}^{n} \sum_{k=0}^{3} \frac{\mathrm{e}^{-m_{j}^{2} /(\mathcal{D}(1+t))}}{(1+t)^{k}} L_{k}^{(-1 / 2)}\left(\frac{m_{j}^{2}}{\mathcal{D}(1+t)}\right)\right) \frac{d t}{(1+t)^{n / 2}}
$$

for the integer vectors $\mathbf{m}=\left(m_{1}, \ldots, m_{n}\right) \in[-240,240]^{n}$, which reduces by a quadrature

$$
I_{3}\left(\frac{\mathbf{m}}{\sqrt{\mathcal{D}}}\right) \approx \sum_{\ell=1}^{R} \frac{\omega_{\ell}}{\left(1+\tau_{\ell}\right)^{n / 2}} \prod_{j=1}^{n} \sum_{k=0}^{3} \frac{\mathrm{e}^{-m_{j}^{2} /\left(\mathcal{D}\left(1+\tau_{\ell}\right)\right)}}{\left(1+\tau_{\ell}\right)^{k}} L_{k}^{(-1 / 2)}\left(\frac{m_{j}^{2}}{\mathcal{D}\left(1+\tau_{\ell}\right)}\right)
$$

to the computation of one vector of length 481 with the components

$$
\sum_{k=0}^{M-1} \frac{\mathrm{e}^{-m^{2} /\left(\mathcal{D}\left(1+\tau_{\ell}\right)\right)}}{\left(1+\tau_{\ell}\right)^{k}} L_{k}^{(-1 / 2)}\left(\frac{m^{2}}{\mathcal{D}\left(1+\tau_{\ell}\right)}\right), \quad m=-240, \ldots, 240,
$$

for any quadrature node $\tau_{\ell}$ and $M=4$. Let us mention, that for all calculations, reported here and in Table 2 below, the same quadrature rule is used for computing the integrals $I_{3}(\mathbf{m} / \sqrt{\mathcal{D}})$.

Table 2 shows that the cubature method is effective also for much higher space dimensions. We compare the exact values of $\mathcal{L}_{n} u_{2}$ and the approximate values $\mathcal{L}_{4, h}^{(n)} u_{2}$ at the same grid points $\left(x_{1}, 0, \ldots, 0\right) \in \mathbb{R}^{n}$ for space dimensions $n=10^{5}, 10^{6}, 2 \cdot 10^{6}$. The parameters of $\mathcal{L}_{4, h}^{(n)}$ are $\mathcal{D}=3.5$ and $h=0.025$.

TABLE 2. Exact values of $\mathcal{L}_{n} u_{2}\left(x_{1}, 0, \ldots, 0\right)$, absolute and relative errors using $\mathcal{L}_{4,0.025}^{(n)}$

\begin{tabular}{r|c|cc|cc|cc}
\hline$n$ & & 10000 & & 100000 & & 200000 & \\
\hline$x_{1}$ & exact & abs. error & rel. error & abs. error & rel. error & abs. error & rel. error \\
\hline 0.0 & $-1.0000 \mathrm{E}-00$ & $5.88 \mathrm{E}-05$ & $5.88 \mathrm{E}-05$ & $2.04 \mathrm{E}-03$ & $2.04 \mathrm{E}-03$ & $2.15 \mathrm{E}-03$ & $2.15 \mathrm{E}-03$ \\
1.0 & $-3.6788 \mathrm{E}-01$ & $2.16 \mathrm{E}-05$ & $5.87 \mathrm{E}-05$ & $7.51 \mathrm{E}-04$ & $2.04 \mathrm{E}-03$ & $7.92 \mathrm{E}-04$ & $2.15 \mathrm{E}-03$ \\
2.0 & $-1.8316 \mathrm{E}-02$ & $1.08 \mathrm{E}-06$ & $5.88 \mathrm{E}-05$ & $3.74 \mathrm{E}-05$ & $2.04 \mathrm{E}-03$ & $3.94 \mathrm{E}-05$ & $2.15 \mathrm{E}-03$ \\
3.0 & $-1.2341 \mathrm{E}-04$ & $7.34 \mathrm{E}-09$ & $5.95 \mathrm{E}-05$ & $2.52 \mathrm{E}-07$ & $2.04 \mathrm{E}-03$ & $2.66 \mathrm{E}-07$ & $2.15 \mathrm{E}-03$ \\
4.0 & $-1.1254 \mathrm{E}-05$ & $6.96 \mathrm{E}-12$ & $6.18 \mathrm{E}-05$ & $2.31 \mathrm{E}-10$ & $2.05 \mathrm{E}-03$ & $2.43 \mathrm{E}-10$ & $2.16 \mathrm{E}-03$ \\
5.0 & $-1.3888 \mathrm{E}-11$ & $9.30 \mathrm{E}-16$ & $6.70 \mathrm{E}-05$ & $2.86 \mathrm{E}-14$ & $2.06 \mathrm{E}-03$ & $3.01 \mathrm{E}-14$ & $2.17 \mathrm{E}-03$ \\
\hline
\end{tabular}

The computing times on a Xeon processor with $3.4 \mathrm{GHz}$ are 0.42 seconds for space dimension $n=10000,5.12$ seconds for $n=100000$, and 11.63 seconds for $n=200000$.

In Table 3 we report on the absolute errors and the approximation rates for the Newton potential $\mathcal{L}_{n} u_{2}(1,0, \ldots, 0)=-0.3678794411714423$ in the space dimensions $n=3,10,500,2000$, and 30000 . The approximate values are computed by the cubature formulas $\mathcal{L}_{M, h}^{(n)}$ defined by (3.4) for $M=1,2,3,4$, having the approximation orders (2.6), with $\mathcal{D}=5$. We use uniform grids of size $h=0.1 \cdot 2^{1-k}$, $k=0, \ldots, 4$, i.e., on the finest grid one has to compute vectors of length 1921 with the components (4.1) for $m=-960, \ldots, 960$.

The numerical results show that the high order cubature formulas give essentially better approximations of the Newton potential than second order formulas. For very high dimensional cases the second order formula fails, whereas the eighth order formula $\mathcal{L}_{4, h}^{(n)}$ approximates with the predicted approximation rate. 
TABLE 3. Absolute errors and approximation rates for $\mathcal{L}_{n} u_{2}(1,0, \ldots, 0)$ using $\mathcal{L}_{M, h}^{(n)}$

\begin{tabular}{|c|c|c|c|c|c|c|c|c|c|c|}
\hline \multicolumn{11}{|c|}{$M=4$} \\
\hline$n$ & 3 & & 10 & & 500 & & 2000 & & 30000 & \\
\hline$h^{-1}$ & error & rate & error & rate & error & rate & error & rate & error & rate \\
\hline 5 & $4.99 \mathrm{E}-05$ & & $6.33 \mathrm{E}-04$ & & $3.93 \mathrm{E}-02$ & & $1.34 \mathrm{E}-01$ & & $3.67 \mathrm{E}-01$ & \\
\hline 10 & $4.73 \mathrm{E}-07$ & 6.72 & $4.16 \mathrm{E}-06$ & 7.25 & $2.62 \mathrm{E}-04$ & 7.22 & $1.05 \mathrm{E}-03$ & 6.99 & $1.55 \mathrm{E}-02$ & 4.57 \\
\hline 20 & $2.32 \mathrm{E}-09$ & 7.67 & $1.88 \mathrm{E}-08$ & 7.79 & $1.17 \mathrm{E}-06$ & 7.81 & $4.69 \mathrm{E}-06$ & 7.81 & $7.04 \mathrm{E}-05$ & 7.78 \\
\hline 40 & $9.64 \mathrm{E}-12$ & 7.91 & $7.64 \mathrm{E}-11$ & 7.94 & $4.75 \mathrm{E}-09$ & 7.94 & $1.91 \mathrm{E}-08$ & 7.94 & $2.86 \mathrm{E}-07$ & 7.94 \\
\hline 80 & $4.99 \mathrm{E}-14$ & 7.60 & $4.02 \mathrm{E}-13$ & 7.57 & $2.50 \mathrm{E}-11$ & 7.57 & $1.00 \mathrm{E}-10$ & 7.57 & $1.51 \mathrm{E}-09$ & 7.57 \\
\hline \multicolumn{11}{|c|}{$M=3$} \\
\hline 5 & $1.45 \mathrm{E}-04$ & & $4.11 \mathrm{E}-03$ & & $1.98 \mathrm{E}-01$ & & $3.51 \mathrm{E}-01$ & & $3.68 \mathrm{E}-01$ & \\
\hline 10 & $5.05 \mathrm{E}-06$ & 4.84 & $9.35 \mathrm{E}-05$ & 5.46 & $6.23 \mathrm{E}-03$ & 4.99 & $2.44 \mathrm{E}-02$ & 3.85 & $2.37 \mathrm{E}-01$ & 0.64 \\
\hline 20 & $9.76 \mathrm{E}-08$ & 5.69 & $1.62 \mathrm{E}-06$ & 5.85 & $1.08 \mathrm{E}-04$ & 5.85 & $4.34 \mathrm{E}-04$ & 5.81 & $6.46 \mathrm{E}-03$ & 5.19 \\
\hline 40 & $1.61 \mathrm{E}-09$ & 5.92 & $2.60 \mathrm{E}-08$ & 5.96 & $1.73 \mathrm{E}-06$ & 5.96 & $6.95 \mathrm{E}-06$ & 5.96 & $1.04 \mathrm{E}-04$ & 5.95 \\
\hline 80 & $2.55 \mathrm{E}-11$ & 5.98 & $4.09 \mathrm{E}-10$ & 5.99 & $2.72 \mathrm{E}-08$ & 5.99 & $1.09 \mathrm{E}-07$ & 5.99 & $1.64 \mathrm{E}-06$ & 5.99 \\
\hline \multicolumn{11}{|c|}{$M=2$} \\
\hline 5 & $1.43 \mathrm{E}-03$ & & $2.89 \mathrm{E}-02$ & & $3.66 \mathrm{E}-01$ & & $3.68 \mathrm{E}-01$ & & $3.68 \mathrm{E}-01$ & \\
\hline 10 & $1.04 \mathrm{E}-04$ & 3.78 & $2.32 \mathrm{E}-03$ & 3.64 & $1.29 \mathrm{E}-01$ & 1.51 & $3.02 \mathrm{E}-01$ & 0.28 & $3.68 \mathrm{E}-01$ & 0.00 \\
\hline 20 & $6.99 \mathrm{E}-06$ & 3.90 & $1.55 \mathrm{E}-04$ & 3.91 & $1.04 \mathrm{E}-02$ & 3.63 & $3.98 \mathrm{E}-02$ & 2.92 & $3.02 \mathrm{E}-01$ & 0.28 \\
\hline 40 & $4.46 \mathrm{E}-07$ & 3.97 & $9.83 \mathrm{E}-06$ & 3.98 & $6.66 \mathrm{E}-04$ & 3.96 & $2.67 \mathrm{E}-03$ & 3.90 & $3.81 \mathrm{E}-02$ & 2.99 \\
\hline 80 & $2.80 \mathrm{E}-08$ & 3.99 & $6.17 \mathrm{E}-07$ & 3.99 & $4.18 \mathrm{E}-05$ & 3.99 & $1.68 \mathrm{E}-04$ & 3.99 & $2.51 \mathrm{E}-03$ & 3.92 \\
\hline \multicolumn{11}{|c|}{$M=1$} \\
\hline 5 & $3.73 \mathrm{E}-02$ & & $1.93 \mathrm{E}-01$ & & $3.68 \mathrm{E}-01$ & & $3.68 \mathrm{E}-01$ & & $3.68 \mathrm{E}-01$ & \\
\hline 10 & $9.29 \mathrm{E}-03$ & 2.00 & $6.56 \mathrm{E}-02$ & 1.56 & $3.68 \mathrm{E}-01$ & 0.00 & $3.68 \mathrm{E}-01$ & 0.00 & $3.68 \mathrm{E}-01$ & 0.00 \\
\hline 20 & $2.31 \mathrm{E}-03$ & 2.01 & $1.79 \mathrm{E}-02$ & 1.88 & $3.51 \mathrm{E}-01$ & 0.07 & $3.68 \mathrm{E}-01$ & 0.00 & $3.68 \mathrm{E}-01$ & 0.00 \\
\hline 40 & $5.75 \mathrm{E}-04$ & 2.00 & $4.56 \mathrm{E}-03$ & 1.97 & $1.99 \mathrm{E}-01$ & 0.82 & $3.52 \mathrm{E}-01$ & 0.07 & $3.68 \mathrm{E}-01$ & 0.00 \\
\hline 80 & $1.44 \mathrm{E}-04$ & 2.00 & $1.15 \mathrm{E}-03$ & 1.99 & $6.50 \mathrm{E}-02$ & 1.61 & $1.99 \mathrm{E}-01$ & 0.82 & $3.68 \mathrm{E}-01$ & 0.00 \\
\hline
\end{tabular}

\section{Quadrature RUles FOR INTEGRAL REPRESEntations}

Here we describe the quadrature rule used to get separated representations of the volume applied to the basis functions. We give some numerical results on the quadrature error for second and fourth order formulas approximating the Newton potential and the potential of $-\Delta+a^{2}$.

5.1. Quadratures. It is well known that the classical trapezoidal rule is exponentially converging for certain classes of integrands, for example, periodic functions or rapidly decaying functions on the real line. For any sufficiently smooth function, say of the Schwartz class $\mathcal{S}(\mathbb{R})$, Poisson's summation formula yields that

$$
h \sum_{k=-\infty}^{\infty} f(k h)=\sum_{k=-\infty}^{\infty} \hat{f}\left(\frac{2 \pi k}{h}\right) .
$$

Here $\hat{f}$ is the Fourier transform

$$
\hat{f}(\lambda)=\int_{-\infty}^{\infty} f(x) \mathrm{e}^{-2 \pi i x \lambda} d x .
$$


Thus,

$$
\int_{-\infty}^{\infty} f(x) d x-h \sum_{k=-\infty}^{\infty} f(k h)=\sum_{k \neq 0} \hat{f}\left(\frac{2 \pi k}{h}\right)
$$

which indicates that by choosing special substitutions, which transform the integrand to a rapidly decaying function with rapidly decaying Fourier transform, the trapezoidal rule of step size $h$ can provide very accurate approximations of the integral for a relatively small number of quadrature nodes $\{k h\}$.

Additionally, if the integrand is analytic in a strip $D_{d}=\{z \in \mathbb{C}:|\operatorname{Im} z|<d\}$ such that

$$
N\left(f, D_{d}\right)=\|f(\cdot+i d)\|_{L_{1}(\mathbb{R})}+\|f(\cdot-i d)\|_{L_{1}(\mathbb{R})}<\infty,
$$

then results from Sinc approximation can be applied to derive error estimates for the trapezoidal rule. It was shown in 6, 8, that for doubly exponentially decaying $f$, i.e.,

$$
|f(x)| \leq C \exp \left(-a \mathrm{e}^{b|x|}\right) \quad \text { for all } x \in \mathbb{R} \quad \text { with constants } a, b, C>0,
$$

the truncated rule with $h=\log (2 \pi a N / b) /(a N)$ satisfies

$$
\left|\int_{-\infty}^{\infty} f(x) d x-h \sum_{k=-N}^{N} f(k h)\right| \leq C N\left(f, D_{d}\right) e^{-2 \pi a d N / \log (2 \pi a N / b)} .
$$

5.2. Newton potentials. We make the substitutions

$$
t=\mathrm{e}^{\xi}, \quad \xi=a\left(\tau+\mathrm{e}^{\tau}\right) \quad \text { and } \quad \tau=b\left(u-\mathrm{e}^{-u}\right),
$$

with certain positive constants $a, b$, proposed in Waldvogel 15. Then the integrals (3.6) are transformed to integrals over $\mathbb{R}$ with integrands $f(u, \mathbf{x})$ decaying doubly exponentially in $u$. After the substitution we have

$$
I_{1}(\mathbf{x})=a b \int_{-\infty}^{\infty} \frac{\left(1+\mathrm{e}^{-u}\right)\left(1+\exp \left(b\left(u-\mathrm{e}^{-u}\right)\right)\right) \phi(u)}{(1+\phi(u))^{n / 2}} \mathrm{e}^{-|\mathbf{x}|^{2} /(1+\phi(u))} d u
$$

with $\phi(u)=\exp \left(a b\left(u-\mathrm{e}^{-u}\right)+a \exp \left(b\left(u-\mathrm{e}^{-u}\right)\right)\right)$. Similarly,

$$
I_{M}(\mathbf{x})=a b \int_{-\infty}^{\infty} \frac{\left(1+\mathrm{e}^{-u}\right)\left(1+\exp \left(b\left(u-\mathrm{e}^{-u}\right)\right)\right) \phi(u)}{(1+\phi(u))^{n / 2}} \prod_{j=1}^{n} g_{M}\left(u, x_{j}\right) d u
$$

with the function

$$
g_{M}(u, x)=\mathrm{e}^{-x^{2} /(1+\phi(u))} \sum_{k=0}^{M-1} \frac{L_{k}^{(-1 / 2)}\left(x^{2} /(1+\phi(u))\right)}{(1+\phi(u))^{k}} .
$$

For the practical application it is important that the quadrature rule

$$
\int_{-\infty}^{\infty} f(u, \mathbf{x}) d u \approx h \sum_{k=N_{0}}^{N_{1}} f(h k, \mathbf{x})
$$

approximates $I_{M}(\mathbf{x})$ with prescribed error uniformly for $|\mathbf{x}| \leq K$ with a minimal number of summands. This number strongly depends on $K$ and on the parameters $a, b$ in the transformation (5.3). For the computations in Section 4, which cover a wide range of dimensions and different orders of the cubature formulas, we did 
not try to find optimal parameter sets. The numbers given in Tables 1 and 2 were obtained with the parameters $a=b=2$ in (5.3) and $h=.02, N_{0}=-35, N_{1}=80$ in (5.4). For the results of Table 3 we have chosen $a=6, b=5$ and $h=.003$, $N_{0}=39, N_{1}=250$. We report in the following on some test to determine optimal parameters for second and fourth order formulas in low dimensional cases (see also [10]).

5.2.1. Approximation to the integral $I_{1}(\mathbf{x})$. We assume in (5.3) $a=b=1$. Figure 1 illustrates the graph of the integrand function $f(u, \mathbf{x}), u \in(-4,4), n=3$, for different values of $|\mathbf{x}| \leq 10^{3}$. A similar behavior holds for other dimensions $n$.
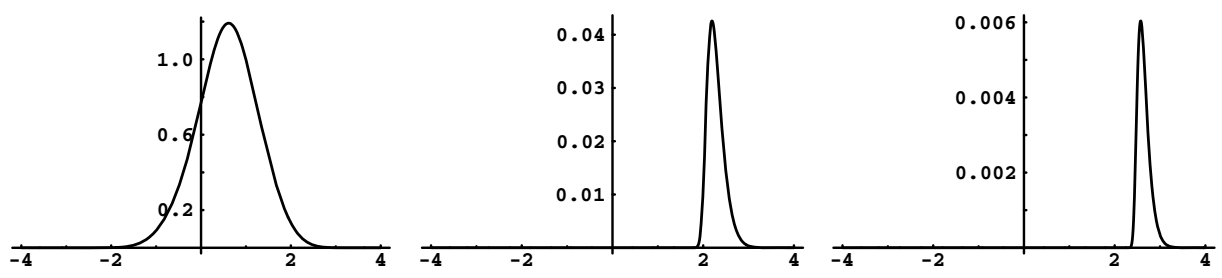

Figure 1. The plot of the integrand function $f(u, \mathbf{x})(a=b=1)$ in $I_{1}(\mathbf{x})$ for $|\mathbf{x}|=0,100,1000$ (from the left to the right) in the interval $u \in(-4,4)$.

Table 4 presents the maximum step $h_{0}$ and the minimum number of quadrature points required to achieve the relative error $\epsilon$, uniformly in $|\mathbf{x}| \in\left[0,10^{3}\right]$ for space dimensions $n=3,4,5,6$.

TABLE 4. The approximation of $I_{1}(\mathbf{x})$ for $|\mathbf{x}| \leq 10^{3}$, with $a=b=1$ in $(5.3)$

\begin{tabular}{|c|c|c|c|c|c|c|c|c|}
\hline & \multicolumn{2}{|l|}{$n=3$} & \multicolumn{2}{|l|}{$n=4$} & \multicolumn{2}{|l|}{$n=5$} & \multicolumn{2}{|l|}{$n=6$} \\
\hline rel. error & $h_{0}$ & nodes & $h_{0}$ & nodes & $h_{0}$ & nodes & $h_{0}$ & nodes \\
\hline $1 \mathrm{E}-05$ & 0.072 & 82 & 0.072 & 77 & 0.065 & 83 & 0.059 & 90 \\
\hline $1 \mathrm{E}-07$ & 0.055 & 116 & 0.051 & 121 & 0.060 & 96 & 0.044 & 130 \\
\hline $1 \mathrm{E}-09$ & 0.043 & 161 & 0.040 & 164 & 0.037 & 169 & 0.035 & 178 \\
\hline $1 \mathrm{E}-11$ & 0.036 & 205 & 0.033 & 206 & 0.033 & 200 & 0.029 & 220 \\
\hline
\end{tabular}

It is possible to play with different parameters $a$ and $b$ in order to diminish the number of summands in the quadrature formula. Consider, e.g., the case $a=6$ and $b=5$. Figure 2 shows the graph of $f(u,|\mathbf{x}|), u \in(0,0.85)$ for different values of $|\mathbf{x}|$. The numerical results for this quadrature are given in Table 5 .

TABLE 5. The approximation of $I_{1}(\mathbf{x})$ for $|\mathbf{x}| \leq 10^{3}$, with $a=$ $6, b=5$ in (5.3)

\begin{tabular}{c|cc|cc|cc|cc}
\hline & $n=3$ & & $n=4$ & & $n=5$ & & $n=6$ & \\
\hline rel. error & $h_{0}$ & nodes & $h_{0}$ & nodes & $h_{0}$ & nodes & $h_{0}$ & nodes \\
\hline $1 \mathrm{E}-05$ & 0.0077 & 61 & 0.0070 & 83 & 0.0069 & 57 & 0.0058 & 70 \\
$1 \mathrm{E}-07$ & 0.0055 & 111 & 0.0049 & 96 & 0.0046 & 112 & 0.0042 & 117 \\
$1 \mathrm{E}-09$ & 0.0042 & 170 & 0.0037 & 169 & 0.0034 & 179 & 0.0037 & 158 \\
$1 \mathrm{E}-11$ & 0.0034 & 247 & 0.0033 & 200 & 0.0031 & 221 & 0.0028 & 242 \\
\hline
\end{tabular}



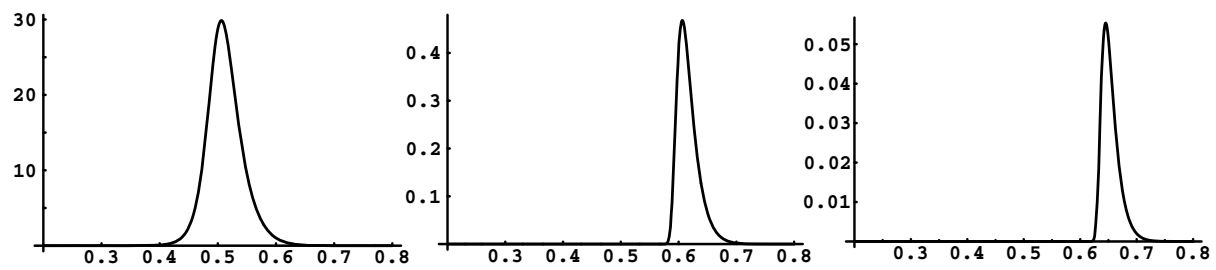

Figure 2. The plot of the integrand function $f(u, \mathbf{x})(a=6, b=$ $5)$ in $I_{1}(\mathbf{x})$ for $|\mathbf{x}|=0,100,1000$ (from the left to the right) in the interval $u \in(0,0.85)$.

5.2.2. Approximation to the integral $I_{2}(\mathbf{x})$. Next, we discuss the computation of the integral

$$
I_{2}(\mathbf{x})=\int_{0}^{\infty} \prod_{j=1}^{n} \mathrm{e}^{-x_{j}^{2} /(t+1)}\left(\frac{3+2 t}{2(1+t)}-\frac{x_{j}^{2}}{(1+t)^{2}}\right) \frac{d t}{(1+t)^{n / 2}}
$$

using the variable transformation (5.3) and the trapezoidal rule (5.4), for $n=3$ and $n=4$. The graphs of the integrands $f(u, \mathbf{x})$ are very similar to the case of $I_{1}(\mathbf{x})$. In the numerical results, for the sake of simplicity, we assumed $\mathbf{x}=(x, x, x)$, with $|\mathbf{x}| \leq 10^{3}$. The maximum step $h_{0}$ and the minimum number of quadrature points required to achieve the relative error $\epsilon$ uniformly in $|\mathbf{x}| \in\left[0,10^{3}\right]$ are presented in Table 6 for the cases $a=b=1$ and $a=6, b=5$.

TABLE 6 . The approximation of $I_{2}(\mathbf{x})$ for $|\mathbf{x}| \leq 10^{3}$, with $a=b=1$ (left) and $a=6, b=5$ (right)

\begin{tabular}{|c|c|c|c|c|c|c|c|c|}
\hline & \multicolumn{2}{|l|}{$n=3$} & \multicolumn{2}{|l|}{$n=4$} & \multicolumn{2}{|l|}{$n=3$} & \multicolumn{2}{|l|}{$n=4$} \\
\hline rel. error & $h_{0}$ & nodes & $h_{0}$ & nodes & $h_{0}$ & nodes & $h_{0}$ & nodes \\
\hline $1 \mathrm{E}-05$ & 0.072 & 82 & 0.072 & 77 & 0.0077 & 63 & 0.0074 & 57 \\
\hline $1 \mathrm{E}-07$ & 0.055 & 118 & 0.051 & 121 & 0.0052 & 114 & 0.0046 & 120 \\
\hline $1 \mathrm{E}-09$ & 0.043 & 163 & 0.040 & 163 & 0.0042 & 175 & 0.0037 & 175 \\
\hline $1 \mathrm{E}-11$ & 0.036 & 204 & 0.033 & 206 & 0.0034 & 234 & 0.0033 & 222 \\
\hline
\end{tabular}

5.3. Potentials of advection-diffusion operators. We consider the special case $\mathbf{b}=0, c=a^{2}>0$, which leads to the one-dimensional representation of the volume potential applied to $\prod \widetilde{\eta}_{2 M}$,

$$
K_{M}(\mathbf{x})=\int_{0}^{\infty} \mathrm{e}^{-a^{2} t / 4} \prod_{j=1}^{n}\left(\sum_{k=0}^{M-1} \frac{\mathrm{e}^{-x_{j}^{2} /(1+t)}}{(1+t)^{k}} L_{k}^{(-1 / 2)}\left(\frac{x_{j}^{2}}{1+t}\right)\right) \frac{d t}{(1+t)^{n / 2}}, \quad M \geq 1
$$

(cf. (3.8), (3.9)). To get doubly exponential integrands we make the substitution

$$
t=\phi(u) \quad \text { with } \phi(u)=\exp (b(u-\exp (-u)), b>0,
$$

and apply the trapezoidal rule to

$$
b \int_{-\infty}^{\infty} \frac{\mathrm{e}^{-a^{2} \phi(u) / 4}\left(1+\mathrm{e}^{-u}\right) \phi(u)}{(1+\phi(u))^{n / 2}} \mathrm{e}^{-|\mathbf{x}|^{2} /(1+\phi(u))} \prod_{j=1}^{n} \sum_{k=0}^{M-1} \frac{L_{k}^{(-1 / 2)}\left(x_{j}^{2} /(1+\phi(u))\right)}{(1+\phi(u))^{k}} d u .
$$


Figures 3 and 4 illustrate the graph of the integrand $f(u, \mathbf{x})$ of $K_{1}(\mathbf{x}), n=3$, for different values of $|\mathbf{x}|$ and $a^{2}=0.01$ and $a^{2}=4$.
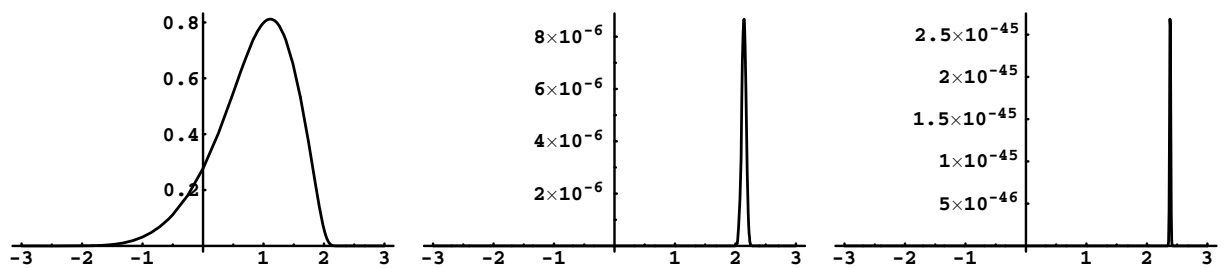

Figure 3. The plot of the integrand function $f(u, \mathbf{x})$ in $K_{1}(\mathbf{x}), a^{2}=0.01, b=1$ for $|\mathbf{x}|=0,100,1000$ (from the left to the right) in the interval $u \in(-3,3)$.
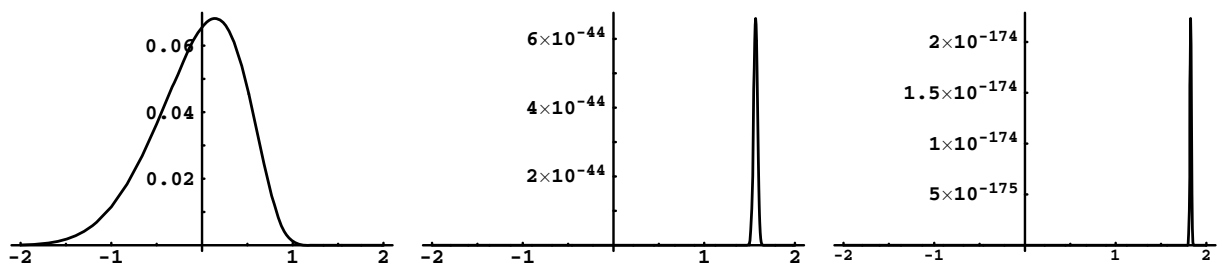

Figure 4. The plot of the integrand function $f(u, \mathbf{x})$ in $K_{1}(\mathbf{x}), a^{2}=4, b=1$ for $|\mathbf{x}|=0,50,200$ (from the left to the right) in the interval $u \in(-2,2)$.

In Table 7 we present the maximum step $h_{0}$ and the minimum number of quadrature points in formula (5.4) for the integral $K_{1}(\mathbf{x})$ for $n=3$, in the cases $a^{2}=0.01,0.1,1,4$. We have chosen $b=1$ in the substitution (5.5).

TABLE 7 . The approximation of $K_{1}(\mathbf{x})$ with different values of $a^{2}$ for $|\mathbf{x}| \leq 10^{3}$

\begin{tabular}{c|cc|cc|cc|cc}
\hline$a^{2}$ & & 0.01 & & 0.1 & & 1 & & 4 \\
\hline rel. error & $h_{0}$ & nodes & $h_{0}$ & nodes & $h_{0}$ & nodes & $h_{0}$ & nodes \\
\hline $1 \mathrm{E}-05$ & 0.58 & 20 & 0.58 & 17 & 0.48 & 15 & 0.44 & 13 \\
$1 \mathrm{E}-07$ & 0.47 & 25 & 0.42 & 16 & 0.38 & 20 & 0.36 & 17 \\
$1 \mathrm{E}-09$ & 0.39 & 32 & 0.40 & 25 & 0.37 & 22 & 0.31 & 21 \\
$1 \mathrm{E}-11$ & 0.30 & 43 & 0.29 & 36 & 0.29 & 28 & 0.27 & 25 \\
$1 \mathrm{E}-13$ & 0.26 & 50 & 0.25 & 43 & 0.25 & 34 & 0.25 & 29 \\
\hline
\end{tabular}

\section{ACKNOWLEDGMENTS}

The authors would like to thank B. Khoromskij for valuable discussions concerning fast computations of high dimensional problems. 


\section{REFERENCES}

1. M. Abramowitz and I. A. Stegun, Handbook of Mathematical Functions, Dover Publ., New York, 1968.

2. G. Beylkin, R. Cramer, G. Fann, and R. J. Harrison, Multiresolution separated representations of singular and weakly singular operators, Appl. Comput. Harmon. Anal. 23 (2007), 235-253. MR2344613 (2008m:65356)

3. G. Beylkin and M. J. Mohlenkamp, Numerical operator calculus in higher dimensions, Proc. Nat. Acad. Sci. USA 99 (2002), 10246-10251. MR.1918798 (2003h:65071)

4. G. Beylkin and M. J. Mohlenkamp, Algorithms for numerical analysis in high dimensions, SIAM J. Sci. Comput. 26, 6 (2005), 2133-2159. MR2196592(2006j:65022)

5. C. Bertoglio and B. N. Khoromskij, Low rank tensor-product approximation of projected Green kernels via sinc-quadratures, Preprint 79, MPI MiS 2008.

6. I. P. Gavrilyuk, W. Hackbusch, and B. N. Khoromskij, Hierarchical tensor-product approximation of the inverse and related operators in high-dimensional elliptic problems, Computing 74 (2005), 131-157. MR2133692 (2006f:65049)

7. W. Hackbusch, Efficient convolution with the Newton potential in $d$ dimensions, Numer. Math. 110 (2008), 449-489. MR 2447246 (2009i:44006)

8. W. Hackbusch and B. N. Khoromskij, Tensor-product approximation to multidimensional integral operators and Green's functions, SIAM J. Matrix Anal. Appl. 30, 3 (2008), 12331253. MR 2447450 (2009g:45023)

9. B. N. Khoromskij, Fast and accurate tensor approximation of multivariate convolution with linear scaling in dimension, Preprint 36, MPI MiS 2008: J. Comp. Appl. Math., 2010, DOI:10.1016/j.cam.2010.02.004, to appear.

10. F. Lanzara, V. Maz'ya, and G. Schmidt, Tensor product approximations of high dimensional potentials, Preprint 1403, WIAS Berlin 2009.

11. V. Maz'ya, Approximate approximations, in The Mathematics of Finite Elements and Applications. Highlights 1993, J. R. Whiteman, ed., Wiley \& Sons, Chichester, 1994, 77-104. MR.1291219 (95g:65137)

12. V. Maz'ya and G. Schmidt "Approximate Approximations" and the cubature of potentials, Rend. Mat. Acc. Lincei, 6 (1995), s. 9, 161-184. MR1363785 (96i:65017)

13. V. Maz'ya and G. Schmidt, Approximate Approximations, Math. Surveys and Monographs vol. 141, AMS 2007.

14. V. Maz'ya and G. Schmidt, Potentials of Gaussians and approximate wavelets, Math. Nachr. 280 (2007), no. 9-10, 1176-1189. MR.2334668 (2009b:65062)

15. J. Waldvogel, Towards a general error theory of the trapezoidal rule. Approximation and Computation 2008. Nis, Serbia, August 25-29, 2008:

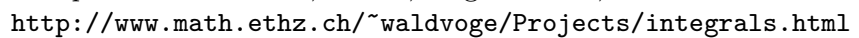

Dipartimento di Matematica, Università "La Sapienza", Piazzale Aldo Moro 2, 00185 Rome, ITALY

E-mail address: lanzara@mat.uniroma1.it

Department of Mathematical Sciences, M\&O Building, University of Liverpool, Liverpool L69 3BX, United Kingdom -And- Department of Mathematics, University of LiNKÖPING, 58183 LiNKÖPING, SWEDEN

E-mail address: vlmaz@mai.liu.se

Weierstrass Institute for Applied Analysis and Stochastics, Mohrenstr. 39, 10117 BerLin, Germany

E-mail address: schmidt@wias-berlin.de 